\title{
inalco PRESSES
}

Transmettre à tous, diffuser plus loin

\author{
RÉSUMÉ DE THÈSE
} La puissance des genres fictionnels de l'imaginaire,
sociologie d'une mouvance sociétale

\author{
Frédérique RÉGUANT
}

Sociétés Plurielles, $\mathbf{n}^{\circ} 3$
Varia

Les Presses de l'Inalco publient des ouvrages scientifiques et des revues qui associent aires culturelles et champs disciplinaires.

EXIGENCE DE QUALITÉ avec des évaluations en double aveugle ;

Open Access : diffusion internationale et ouvrages toujours disponibles;

LICENCES D'ÉDITION SOUS CREATIVE COMMONS pour protéger les auteurs et leurs droits;

Publications multisupports et ENRICHISSEMENTS sémantiques et audio-visuels;

MÉTADONNÉES MULTILINGUES : titres, résumés, mots-clés.
L'offre éditoriale s'organise autour de collections aires géographiques (AsieS, EuropeS, AfriqueS, MéditerranéeS, TransAireS, AmériqueS, OcéanieS) et de séries correspondant à des regroupements disciplinaires (langues et linguistique, sciences humaines et sociales, arts et lettres, sciences politiques, économiques et juridiques, oralité, traduction).

Les Presses de l'Inalco éditent de nombreuses revues: Cabiers balkaniques, Cabiers de littérature orale, Cipango, Cipango - Japanese studies, Études océan Indien, Études finno-ougriennes, Mandenkan, Slovo, Sociétés Plurielles, Yod.

https://www.pressesinalco.fr

2, rue de Lille - 75007 Paris 


\title{
Sociétés plurielles
}

\author{
Varia
}

Numéro 3 - Année 2019 


\title{
La puissance des genres fictionnels de l'imaginaire, sociologie d'une mouvance sociétale
}

\author{
Thèse de doctorat en sociologie, sous la direction
} de Patrick Tacussel, soutenue le 28 novembre 2017, Université Paul Valéry - Montpellier III, 520 p.

Frédérique RÉGUANT

En explorant les mutations de notre société, nous avons étudié, durant nos années de doctorat, un nouveau phénomène, celui que nous avons nommé « les années folles » de l'imaginaire. En réponse aux changements, crises et bouleversements dont notre société a fait face, la société et les individus qui la composent se sont tournés vers d'autres mondes, d'autres univers entièrement construits par l'imagination des Hommes. Sous les titres « fantasy », « fantastique » et « science-fiction » un nouvel engouement s'est formé. Depuis une quinzaine d'années, se dresse un constat indéniable : qu'il s'agisse de littérature ou de cinéma, les fictions de l'imaginaire se multiplient, touchant un public de plus en plus étendu. L'importance grandissante des loisirs et leur reconnaissance comme élément primordial de la vie de l'individu sont des phénomènes qui nous ont conduits, en tant que chercheur, à nous interroger sur cette évolution. Des fans d'Harry Potter aux passionnés du Seigneur des Anneaux, des gamers ${ }^{1}$ aux rôlistes ${ }^{2}$, nous avons étudié le nouvel engouement que ces œuvres suscitent au sein de la vie quotidienne des individus. Harry Potter

1. Du terme anglais Game signifiant «jeu », il désigne les individus s'adonnant à cette pratique et notamment celle des jeux vidéo.

2. Individu pratiquant le jeu de rôle ou « JdR ». 
est l'exemple type, son succès a permis à l'imaginaire fictionnel de retrouver une place de choix dans le quotidien des Hommes. À sa suite, de multiples ouvres surfant sur cette même vague merveilleuse firent leur apparition sur le marché littéraire et cinématographique, conduisant les épris à se constituer sous la forme de communautés de fans. Les effets de ces genres sur les visions du monde contemporain, peu examinés dans notre champ disciplinaire, ont ouvert la voie à cette recherche. En s'impliquant à travers une sociologie multiple : de la culture, de la littérature, de l'imaginaire, du quotidien, de la réception, notre recherche s'est centrée sur la place qu'occupe l'imaginaire (en tant que genre) tant au niveau collectif qu'individuel, son rapport au quotidien et l'intensité qu'il provoque. Notre travail de recherche s'est appuyé sur l'étude d'un corpus d'œuvres littéraires reflétant le marché de l'imaginaire, d'une enquête par questionnaire menée sur un échantillon représentatif de la population ${ }^{3}$ française et d'une série d'interview menée sur des fans. Notre projet de thèse a ainsi porté sur les émotions véhiculées par l'imaginaire de notre temps, en liaison avec les besoins sociaux que renouvelle la société actuelle : envie d'aventure, quête d'évasion, d'étrangeté, associées à un besoin de réenchantement du monde social.

Autrefois considérés de manière négative, promesse illusoire d'un monde meilleur, les genres fictionnels de l'imaginaire se sont émancipés. Dans une période de mouvance, ils ont su s'adapter aux diverses évolutions de notre société. L'histoire de cette dernière se caractérise par plusieurs phases, chacune ayant ses propres caractéristiques, ses propres traits. Période prémoderne, où le seul rapport à la réalité connaissable ne pouvait être que théologique. Période moderne, impulsée par les philosophes des lumières où raison et progrès en était les égéries. Puis aujourd'hui, période où l'ère de la modernité semble s'être achevée à l'instant où le mythe du progrès s'est lui-même effondré. Cette nouvelle période socio-historique dans laquelle nous nous situons se caractérise à la fois par la notion d'abandon des idées de progrès et de sens de l'histoire et, à la fois, par une remise en cause du projet de connaissance rationnelle du monde. D'un point de vue sociologique, l'opposition entre modernité et postmodernité est devenue marquante en 1979 à l'écriture du livre La Condition postmoderne de Jean-François Lyotard. Cette opposition s'inscrit dans une oscillation entre le « désenchantement » et le « réenchantement » du monde. Le désenchantement du monde apparaît dans L'Éthique protestante et l'esprit du capitalisme $e^{4}$ à plusieurs reprises. Il désigne la rationalisation de la vie, mise en œuvre

\section{Agée de 15 à 54 ans}

4. Weber Max, L'Éthique protestante et l'esprit du capitalisme, paru en 1904 et 1905 dans les Archive für Sozialwissenschaft und Sozialpolitik. 
La puissance des genres fictionnels de l'imaginaire, sociologie d'une mouvance sociale

par la révolution industrielle, il est propre à la modernité. Désenchanter c'est chasser la magie et rationaliser. Ce phénomène coïncide avec le recul de la vision du monde primitif, propre à la magie, qui concevait l'univers comme une réalité habitée par des esprits, où s'exerçait la puissance d'individus dotés de pouvoirs extraordinaires 5 . Le processus de rationalisation reste toutefois insatisfaisant. En effet, la science ne peut donner un « sens à la vie » en donnant des principes d'existence aux hommes comme le faisait la religion. Suite au désenchantement du monde, caractéristique de la société moderne, se succéderait donc un réenchantement du monde faisant référence à la nouvelle période dans laquelle baigne notre société. L'homme était pensé comme un être entièrement fait de raison, ce qui n'est pas le cas, il se relie au monde par son imaginaire, son désir, sa sensibilité, se nourrissant de rêves autant que d'explication logiques, de poésies et de légendes.

Carl Gustav Jung en avait donné l'explication il y a un demi-siècle :

l'être humain a autant besoin de raison que d'émotion, de science que de mythe, d'arguments que de symboles ${ }^{6}$.

C'est ici même que réside le paradoxe de « l'ultra modernité » : plus la science progresse, plus nous avons besoin de mythes, d'abandon de rationalisation. D'après Charles Nodier, la modernité et le progrès ne sont que des machines ayant pour but d'éradiquer toute forme de singularité. Dans cette optique, le repli et l'évasion vers l'imaginaire n'est pas pensé en tant que fuite. Certes, « le penchant au merveilleux demeure peut-être la seule compensation vraiment providentielle des misères inséparables de la vie sociale ${ }^{7} \gg$, mais, il est surtout, synonyme de retour euphorique vers l'insouciance : «Grâces soient rendues à Musoeus, à Tieck, à Hoffmann dont les heureux caprices [...] renouvellent, pour les vieux jours de notre décrépitude, les fraîches et brillantes illusions de notre berceau [...], c'est la fontaine de jouvence de l'imagination ${ }^{8}$. » Après l'ère moderne, empreinte de science et de progrès, la domination de la raison semble s'éteindre entrainant avec elle un bouleversement. La société au sein de laquelle nous évoluons aujourd'hui est faite d'affect, de sentiments. C'est ici que nous arrivons au fondement même de notre recherche : les plus grands succès littéraires et cinématographiques de la dernière décennie ont un point commun, ils répondent à notre besoin de merveilleux. Dans un monde

5. Rossi, 1995 , p. 87.

6. LENOIR, 2005.

7. NODIER, 1879.

8. Ibid. 
en constante évolution, où tout va toujours plus vite, les genres fictionnels de l'imaginaire représenteraient une échappatoire, un espace hors du temps (mais utilisé paradoxalement dans le temps présent), où l'homme changerait de « peau », deviendrait qui il souhaiterait être et se remplirait d'émotions diverses. Ayant pris de grandes distances avec la religion, les individus en quête de l'ailleurs, en recherche d'autres univers et de fortes émotions vont se ressourcer dans ces œuvres. Nous assistons à un retour du refoulé, un réenchantement du monde car celui-ci a été désenchanté.

Hérö̈sme, magie, amour, violence, surnaturel, représentent les points d'ancrages de ces différents genres. Le fantastique provoque un sentiment de peur; la science-fiction fait émerger une forme de réflexion et la fantasy émerveille. Grâce aux romans et aux images (cinématographiques et séries télévisuelles), les actes imaginaires ont pris sens. Le cinéma a permis de mettre en image le fabuleux conté dans les romans. Il a offert « aux yeux et au cœur des hommes de tous pays et de toutes cultures les grands archétypes du récit "éternel" racontés de façon telle que chacun peut s'y reconnaître et s'y retrouver aussitôt ${ }^{9}{ }^{\text {} \gg . ~ L e s ~ g e n r e s ~ d e ~ l ' i m a g i n a i r e ~}$ permettent évasion, émotions, dans un univers plus ou moins magique. Les héros nous ressemblent, c'est ce qui permet au public de s'insérer pleinement et de ressentir profondément. Pour Edgar Morin ${ }^{10}$, l'homme possède deux polarités, il y a l'Homo sapiens (l'homme doté de raison) et Homo demens (l'homme de délire, la folie n'est pas maladive, elle apparaît dans la démesure, la colère, la passion incontrôlée). L'individu vit entre ses deux axes et, dans chacun d'eux, il y a l'affectivité, le sentiment, même les actes les plus rationnels mobilisent le centre émotionnel du cerveau. Il n'y a pas de raison sans émotions. Dans ces fictions fusionnent le réel et l'irréel. Cette idée d'ancrage dans la réalité, va créer cet intense aspect séducteur. L'individu a besoin de (re)connaître notre réel à travers l'irréel pour s'y immerger pleinement et ressentir cette intensité émotionnelle. Il ne s'agit pas d'une fuite, l'usager quitte la réalité sans (corporellement) la quitter, seul son esprit vagabonde, il ne cherche plus à contrer la vie, à la changer, il s'investit dans une autre, dans d'autres possibles, il cherche à atteindre le bonheur immédiat :

Alors pour « changer le monde » symboliquement, illusoirement, et mieux supporter notre impuissance individuelle (et notre fatigue d'avance à même essayer) devant l'incapacité collective à en détourner les apocalypses annoncées - férocité du capitalisme financier,

9. Lipovetsky \& SERRoy, 2007, p. 333.

10. Morin, 12 décembre 2013. 
inégalités mondiales, désastres écologiques -, on « augmente » la réalité d'autres possibles, bifurcations alternatives comme autant de promesses qui martèlent « c'est possible », on en réinvente le passé et l'avenir, en y privilégiant les « merveilles ${ }^{11}$.

La fiction va permettre d'augmenter les possibles et de les expérimenter. Au sein de ces nouvelles manières de vivre, l'imaginaire, en tant que genre fictionnel, obtient alors une place privilégiée.

\section{Bibliographie}

BEsSON Anne, 2015, Constellations : des mondes fictionnels dans l'imaginaire contemporain, CNRS Éditions, Paris, 560 p.

Rossi P., 1995, « Max Weber : politique et histoire » in Revue Européenne des sciences sociales, Tome XXXIII, N¹01, p. 87.

LenolR Frédéric, 2005, « Faut-il avoir peur d'Harry Potter » in Le monde des religions, éditorial.

Lipovetsky Gilles \& Serroy Jean, 2007, L'Écran global. Culture-médias et cinéma à l'âge hypermoderne, Seuil, Paris, $361 \mathrm{p}$.

Morin Edgar, 12 décembre 2013, « Amour Poésie Sagesse », interview à l'Université de Nantes, https://www.youtube.com/watch?v=dfSFGMBSWvg.

Nodier Charles, 1879, Contes fantastiques, G. Charpentier, Paris, p. 25.

Weber Max, L'Éthique protestante et l'esprit du capitalisme, paru en 1904 et 1905 dans les Archive für Sozialwissenschaft und Sozialpolitik. ?

11. BESSON, 2015, p. 483. 